\title{
Growth, Yield and Quality of Two Globe Artichoke Cultivars as Affected by Gibberellic Acid, Naphthalene Acetic Acid, Benzyle Amino Purine and Seaweed Extract
}

\author{
Gabr, S.M., H.A. Elkhateb, S.H. Brenegi, and R.G. Aly \\ Horticulture Department, Faculty of Agriculture, Damnhour University.
}

\begin{abstract}
Two field experiments were carried out during the two successive seasons (2016 - 2017 and 2017- 2018), in a private Farm, at Seedy Ghazi- Kafr El Dawar, Behiera Governorate, Egypt. The objectives of this work are to evaluate the outcomes of using gibberellic acid (30 and $60 \mathrm{ppm}$ ), naphthalene acetic acid ( 25 and $50 \mathrm{ppm}$ ),6-N benzyl adenine purine (20 and $30 \mathrm{ppm}$ ) and seaweed extract (1 and $2 \mathrm{~g} / \mathrm{L}$ ) on growth, yield and quality of globe artichoke cv. 'French' and 'Balady' under Behiera Governorate environment. The obtained results of the two seasons revealed that French cultivar showed higher mean values of plant height, number of leaves, leaf area, number of branches, fresh and dry weight, nitrogen and protein in leaves. However, Balady cultivar showed high values of number of early head / plant and number of early head /feddan, total head / plant, number of total head /feddan, weight of edible part/ plant and potassium in leaves. Spraying artichoke plant suing gibberellic acid, naphthalene acetic acid, N-6-benzyle amino purine and seaweed extract with all concentrations showed significant effect on the almost vegetative growth characteristics, yield and its components and chemical characteristics of leaves with different magnitude compared to control treatment. In this respect, gibberellic acid was superior in the case of vegetative growth and early yield characteristics. However, seaweed extract and 6-N benzyl adenine purine gave the highest mean values of total yield and chemical characteristics of leaves. The combined treatment of Balady cultivar and either seaweed extract at $2 \mathrm{~g} / \mathrm{L}$ or N-6-benzyle amino purine at $30 \mathrm{ppm}$ recorded the maximum mean values of the studied characters and might be considered as an optimal treatment for the production of high yield and good quality of globe artichoke plants.
\end{abstract}

Key words: Globe Artichoke, Gibberellic Acid, Naphthalene Acetic Acid, Seaweed Extract.

\section{INTRODUCTION}

Globe artichoke (Cynara scolymus L.) is an important vegetable crop, belongs to the Asteraceae family. It is a perennial rosette plant grown throughout the worldwide for its edible and fleshy heads. It is considered as one of the most significant medicinal plant, too. It has important nutritional status related to its high content of phenolic compounds as flavonoids, inulin, fibers and minerals. Phenolic compounds such as cynarin have effect on hepatitis diseases, hyperlipidemia, dropsy, rheumatism and cholesterol metabolism (Mauromicale et al., 2003).

Artichoke is widely cultivated in the Mediterranean region, including Egypt. The total area grown with artichoke in Egypt was 17287 ha, which produced about 323866 tons (FAO, 2018). High yield and quality of artichoke flower heads during the period of December to February, has major encouragement for promoting artichoke plantation area in Egypt. It is known that artichoke cultivars demand to undergo verbalization to shift from vegetative to generative stage which in the cooler regions starts very early (during the autumn). Stem elongation occurs only in the spring (Bucan et al., 2000; Elia et al., 1991; Mauromicale and Lerna, 2000). Probably out- of- season production occurs during warm part of the year; therefore, plants often do not have enough periods of low temperature to induce flowering; hence foliar application of gibberellic acid $\left(\mathrm{GA}_{3}\right)$ could be an alternative mean to solve this problem and extend both the harvest period and production season. Saber et al. (2003) mentioned $\mathrm{GA}_{3}$ is used extensively in Egypt and other countries to increase the growth and yield of many vegetables such as tomatoes, globe artichoke, cabbages and cauliflower. Several reports stated the profound effects of $\mathrm{GA}_{3}$ on globe artichoke, but outcome results are variable (De Malach et al., 1976; El-Baz et al., 1979 and Mauromicale et al., 2003).

Gibberellic acid is known as growth hormone (endogenously and occurs as natural hormone) for growth and development, and added exogenously as plant growth regulator to hasten or accelerate flowering process that subsequently head production in globe artichoke especially during the period from December to February period. Throughout this period, the local market is in need to kind of vegetable at high price and option for export abroad. Additionally, this period is economically interesting for export, because there is no production in most European countries during these months (Abd ElHameid et al., 2008). In order to accelerate the early production of heads and obtain increased benefits from higher prices, hence exogenous gibberellic acid $\left(\mathrm{GA}_{3}\right)$ application is proposed. Application of $\mathrm{GA}_{3}$ is a common practice to achieve earliness in globe artichoke heading (Abd El-Hameid et al., 2008). Numerous of articles concerning effect of $\mathrm{GA}_{3}$ on flowering of globe artichoke exhibited inconsistent trend in annual artichokes production. 
For instance, according to field observations, Schrader and Keith 1997 reported that foliar application of $\mathrm{GA}_{3}$ for perennial artichoke production; increased earliness slightly but failed to achieve the level of uniformity and earliness. Define type of applied growth regulators, rates and application times are effective for accelerating maturity and increasing uniformity of flowering in artichokes grown as annuals from seed.

The use of other plant hormones such as cytokinins and auxins has an effective role in accelerating maturity and increasing yields of many vegetable crops. Flowering, fruit setting and inhibition of abscission are other plant responses under the direct or indirect control of auxins, while many effects on plant development are under the effect of cytokinins, either in conjunction with auxin or another hormone. Recently, Seaweed extracts are utilized as foliar sprays to improve plant growth and yield. Plants treated with seaweed extracts showed similar physiological responses to those treated with plant growth-regulatory substances. However, there is meager information on the effects of these hormones as well as seaweed extracts on artichokes.

Cultivar is another important factor in determining the quantity and quality of a crop. There are many studies showed high discrepancy between artichoke varieties in several characteristics such as early flowering, yield, and quality. Cultivation the local cultivar (Balady) produces heads with poor quality which are not suitable for exportation purposes. Therefore, more attention should be given to promote artichoke flower head production and its quality through choice suitable cultivars.

Therefore, the objectives of this study are to evaluate the using gibberellic acid, naphthalene acetic acid, 6-N benzyl adenine purine and seaweed extract on growth, yield and quality of globe artichoke cv. 'French' and cv. 'Balady' under Behiera Governorate environment.

\section{MATERIAL AND METHODS}

Two field experiments were carried out during the two successive seasons (2016 - 2017 and 20172018), in a private Farm at Seedy Ghazi- Kafr El Dawar, Behiera Governorate, Egypt, under open field condition, using surface irrigation system. Soil samples of $30 \mathrm{~cm}$ depth were collected and analyzed for some soils physical and chemical properties for the experimental site during both seasons according to, Black (1965). Results of soil physio-chemical analyses are shown in Table 1.

The present experiment included 18 treatments which were the combination between the two cultivars (Balady and French) and spraying treatments: Gibberellic acid $\left(\mathrm{GA}_{3}\right)$ at 30 and $60 \mathrm{ppm}$, Naphthalene acetic acid (NNA) at 25 and $50 \mathrm{ppm}$, $\mathrm{N}-6-$-Benzyle amino purine (6BA) at 20 and $30 \mathrm{ppm}$ and seaweed extract at 1 and $2 \mathrm{~g} / \mathrm{L}$ and control (tap water). Stumps cuttings (crown pieces) as a propagation methods were produced from an old crown of a previous globe artichoke plant, cv. Balady and French in both experiments. The stumps were disinfected by the fungicide Topspin M-70 at the rate of $2 \mathrm{~g} / 1$ for 20 minutes, before planting, subsequently they were air dried. Planting took place on August $15^{\text {th }}$ during both seasons of the study. Each stump has approximately constant weight, more or less, ca. $160 \mathrm{~g} /$ stump. Each experimental unit contained 3 rows, $14 \mathrm{~m}$ long, $1 \mathrm{~m}$ width and the intra-row spacing was $75 \mathrm{~cm}$ apart.

Table 1. Some physical and chemical properties of the experimental location during both seasons of experimentation (2016-2017 and 2017-2018).

\begin{tabular}{|c|c|c|c|}
\hline \multirow{2}{*}{ Soil properties } & & \multicolumn{2}{|c|}{ Seasons } \\
\hline & & 2016-2017 & 2017-2018 \\
\hline \multirow{3}{*}{ Particle Size Distribution (\%) } & sand* & 29.5 & 28.9 \\
\hline & silt & 27.6 & 28.8 \\
\hline & clay & 42.9 & 42.3 \\
\hline Textural Class & \multicolumn{2}{|c|}{ Clay Loam } & Clay Loam \\
\hline \multirow{3}{*}{ Chemical } & $\mathrm{pH}$ & 8.55 & 8.39 \\
\hline & $\mathrm{EC}\left(\mathrm{dsm}^{-1}\right)$ & 1.62 & 1.58 \\
\hline & $\mathrm{O} . \mathrm{M}(\%)$ & 1.79 & 1.74 \\
\hline \multirow{7}{*}{ Soluble Ions (meq/L) } & $\mathrm{CaCO}_{3}(\%)$ & 21.4 & 22.6 \\
\hline & $\mathrm{Na}^{+}$ & 5.3 & 5.24 \\
\hline & $\mathrm{Ca}^{++}$ & 5 & 5.1 \\
\hline & $\mathrm{Mg}^{++}$ & 4.8 & 4.9 \\
\hline & $\mathrm{K}^{+}$ & 0.12 & 0.1 \\
\hline & $\mathrm{Cl}^{-}$ & 8.8 & 8.7 \\
\hline & $\mathrm{SO}_{4}^{--}$ & 3.4 & 3.5 \\
\hline
\end{tabular}

*The analyses were carried out at the Agricultural Directorate Lab of Damanhur city, El-Behera governorate, Egypt. 
The experimental unit area was $42 \mathrm{~m}^{2}$. So, the total number of plants/ feddan were 5333 plants.

The suggested concentrations of spraying treatments were applied as a foliar spraying until run-off using a hand sprayer. All precautions and accuracy were followed during weighing, dissolving and spraying. Foliar application was done just before sunset during both seasons, to avoid deterioration caused by effect of high temperatures and other ambient atmosphere on the applied items. Each spraying treatment was applied three times after planting: 60, 80 and 100 days. The untreated control plants were sprayed with tap water similarly. All experimental units received identical levels of nitrogen, phosphorus and potassium fertilizers as well as organic manure $\left(20 \mathrm{~m}^{3} \quad\right.$ FYM/fed.). Ammonium sulphate $(20.5 \% \mathrm{~N})$ at the rate of 300 $\mathrm{kg} / \mathrm{fed}$. was equally divided and side dressed after 8 , 12 and 16 weeks after planting, Calcium super phosphate $\left(15.5 \% \mathrm{P}_{2} \mathrm{O}_{5}\right)$ at the rate of $250 \mathrm{~kg} / \mathrm{fed}$. was base dressed before planting and potassium sulphate at the rate of $100 \mathrm{~kg} / \mathrm{fed}$. was equally divided and side dressed after 8 and 12 weeks of planting. All other agricultural practices were adopted whenever they were necessary and as commonly recommended for the commercial production of globe artichoke. Harvesting started in the first season on December $10^{\text {th }}, 2016$ and continued at seven days intervals until May $1^{\text {st }}$, 2017. Meanwhile, in the second season, the harvesting period extended from November $29^{\text {th }}$, 2017 and continued to April 30 ${ }^{\text {th }}, 2018$.

The layout experiment was split plots system in a Randomized Complete Blocks Design (RCBD) with three replications. The two cultivars' French' and 'Balady' were arranged as the main plots, whereas, each of gibberellic acid, naphthalene acetic acid,6-N benzyl adenine purine and seaweed extract concentrations were considered as the sub- plots. Two guard rows were left between each two adjacent main plots and one guard row was left between each two adjacent sub-plots to protect against side effects. In each experimental unit, a representative sample of random five plants from each plot was randomly taken at 1110 and 115 days from planting in the first and second experiment, respectively. The following growth traits measurements were recorded.

\section{Vegetative growth characteristics}

Plant height $(\mathrm{cm})$, number of leaves per plant, number of shoots per plant, plant fresh weight (gm), Plant dry weight $(\mathrm{gm})$. Leaf area per plant $\left(\mathrm{cm}^{2}\right)$ was calculated using the weight method as used by Fayed (1997).

\section{Head yield and its components}

At the harvest, a random sample of five plant heads from each plot were randomly collected for measuring the following traits:
Early yield was calculated as the number of all harvested heads/plant and head/ feddan for the first 12 pickings. Total yield was calculated as the number of all harvested heads/ plant and head/feddan, and weight of edible from during the season.

\section{Chemical constituents of leaves.}

Total chlorophyll content was measured by a digital chlorophyll meter SPAD-502.Total nitrogen was determined calorimetrically according to Evenhuis and De Waard (1980). Phosphorus was determined using ammonium molybdate stannous chloride method (A.O.A.C, 1992). Potassium was measured using a flame photometer as described by Singh et al. (2005). Protein (\%) was estimated according to A.O.A.C. (1975).

\section{Statistical Analysis}

All obtained data were subjected to analysis of variance according to the design used by the Costate software package (Costate, 2008). Comparisons among the means of different treatments were carried out using Revised Least Significant test at probability 0.05 to verify significant among treatments.

\section{RESULTS AND DISCUSSION \\ Vegetative growth characters:}

Data presented in Tables (2 and 3) indicated that the main effect of artichoke cultivars differed significantly in their vegetative growth in both seasons. French cultivar showed higher mean values of plant height, number of leaves, leaf area, number of branches, fresh and dry weight than those of balady cultivar. The detected differences between the two cultivars could be related to their genetic features. The present results are in agreement with those reported by Ibrahim (1980) who indicated that artichoke cultivars were significantly differed in their plant size, branching number and shape of leaves as well as varied in length. Also, Ibrahim (2009) reported that plant height, number of leave / plant and average leaf area obtained from French and Imperial Star cultivars, recorded the highest values compared to Balady cultivar.

Okasha et al. (1997) reported that significant diligence in plant growth, i.e., Plant height, number of leaves / plant and both leaf length and width were detected among some artichoke cultivars. Moreover, Hammouda et al. (1993) found that the highest number of leaves was obtained from the Romanian strain compared to Balady cultivar. The obtained results are in agreement with those of Gabr and Sarg (1998) and Gabr et al. (2002) on potatoes.

Regarding the main effect of the spraying treatments (gibberellic acid, naphthalene acetic acid, N-6-benzyle amino purine and seaweed extract) on vegetative growth parameters of artichoke plants, the showed results in Tables (2 and 3) illustrated that spraying gibberellic acid, naphthalene acetic acid, 
Table 2: The main average values of some vegetative growth of artichoke plants as affected by cultivars, NNA, GA3, 6BA and Seaweed Extract during the fall season of 2016 and 2017.

\begin{tabular}{|c|c|c|c|c|c|c|c|c|c|c|c|c|c|}
\hline & \multirow[t]{2}{*}{ Treatments } & \multicolumn{2}{|c|}{$\begin{array}{l}\text { Plant height* } \\
\text { (cm) }\end{array}$} & \multicolumn{2}{|c|}{ Number of leaves /plant } & \multicolumn{2}{|c|}{$\begin{array}{c}\text { leaf area /plant } \\
\left(\mathrm{cm}^{2}\right)\end{array}$} & \multicolumn{2}{|c|}{$\begin{array}{c}\text { Number of branches } \\
\text { /plant }\end{array}$} & \multicolumn{2}{|c|}{$\begin{array}{c}\text { Fresh weight/plant } \\
\text { (gm) }\end{array}$} & \multicolumn{2}{|c|}{$\begin{array}{c}\text { Dry weight/plant } \\
\text { (gm) }\end{array}$} \\
\hline & & $1^{\text {st }}$ season & $2^{\text {nd }}$ season & $1^{\text {st }}$ season & $\mathbf{2}^{\text {nd }}$ season & $1_{\text {season }}^{\text {st }}$ & $2^{2^{\text {nd }} \text { season }}$ & $1_{\text {season }}^{\text {st }}$ & $2^{\text {nd }}$ season & $\mathbf{1}^{\text {st }}{ }_{\text {season }}$ & $2^{2^{\text {nd }} \text { season }}$ & $\mathbf{1}^{\text {st }}{ }_{\text {season }}$ & $\mathbf{2}^{\text {nd }}{ }_{\text {season }}$ \\
\hline \multirow{2}{*}{ 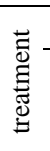 } & French & $115.62 \mathrm{~A}$ & $117.13 \mathrm{~A}$ & $24.17 \mathrm{~A}$ & $24.70 \mathrm{~A}$ & $3016.14 \mathrm{~A}$ & $2837.83 \mathrm{~A}$ & $8.92 \mathrm{~A}$ & $8.92 \mathrm{~A}$ & $3925.50 \mathrm{~A}$ & $3172.9 \mathrm{~A}$ & $1366.06 \mathrm{~A}$ & $1264.85 \mathrm{~A}$ \\
\hline & Balady & $101.22 \mathrm{~B}$ & $108.88 \mathrm{~B}$ & $20.71 \mathrm{~B}$ & $22.29 \mathrm{~B}$ & $2598.26 \mathrm{~B}$ & $2454.38 \mathrm{~B}$ & $8.88 \mathrm{~A}$ & $8.88 \mathrm{~A}$ & $3378.97 \mathrm{~B}$ & $3231.7 \mathrm{~B}$ & $1175.78 \mathrm{~B}$ & $1096.75 \mathrm{~B}$ \\
\hline نั & tap water & $99.33 \mathrm{D}$ & $98.74 \mathrm{~F}$ & $19.00 \mathrm{C}$ & $20.83 \mathrm{~F}$ & $1716.03 \mathrm{D}$ & $1721.33 \mathrm{~F}$ & $7.33 \mathrm{D}$ & $7.33 \mathrm{D}$ & $2015.37 \mathrm{E}$ & 2018.16 I & $514.10 \mathrm{D}$ & $520.35 \mathrm{H}$ \\
\hline \multirow{2}{*}{ 文 } & $25 \mathrm{ppm}$ & $102.00 \mathrm{CD}$ & $105.84 \mathrm{E}$ & $20.00 \mathrm{BC}$ & $22.16 \mathrm{EF}$ & $2253.03 \mathrm{C}$ & $2308.49 \mathrm{E}$ & $7.33 \mathrm{D}$ & $7.33 \mathrm{D}$ & $3112.65 \mathrm{D}$ & $3044.9 \mathrm{H}$ & $1151.68 \mathrm{C}$ & $1138.51 \mathrm{G}$ \\
\hline & $50 \mathrm{ppm}$ & $101.66 \mathrm{CD}$ & $109.34 \mathrm{D}$ & $21.33 \mathrm{ABC}$ & 22.33 DEF & 2913.97 B & $2732.20 \mathrm{C}$ & $8.50 \mathrm{C}$ & $8.50 \mathrm{C}$ & $3370.92 \mathrm{C}$ & $3296.84 \mathrm{~F}$ & $1182.29 \mathrm{C}$ & $1167.60 \mathrm{~F}$ \\
\hline \multirow{2}{*}{ 芯 } & $30 \mathrm{ppm}$ & 105.83BCD & $114.15 \mathrm{C}$ & $20.17 \mathrm{BC}$ & $21.83 \mathrm{EF}$ & 2944.43 B & $2683.93 \mathrm{D}$ & $8.83 \mathrm{BC}$ & $8.83 \mathrm{BC}$ & $3144.08 \mathrm{D}$ & $3142.3 \mathrm{G}$ & $1168.31 \mathrm{C}$ & $1381.50 \mathrm{~B}$ \\
\hline & $60 \mathrm{ppm}$ & $121.50 \mathrm{~A}$ & $121.12 \mathrm{AB}$ & $24.50 \mathrm{AB}$ & $24.66 \mathrm{~B}$ & $2717.73 \mathrm{~B}$ & $2963.88 \mathrm{~A}$ & $10.00 \mathrm{~A}$ & $10.00 \mathrm{~A}$ & $3553.94 \mathrm{C}$ & $3700.8 \mathrm{D}$ & $1304.19 \mathrm{~B}$ & $1184.30 \mathrm{E}$ \\
\hline \multirow{2}{*}{ 苂 } & $20 \mathrm{ppm}$ & $109.50 \mathrm{~B}$ & $115.34 \mathrm{C}$ & $23.83 \mathrm{AB}$ & 23.00CDE & $2859.32 \mathrm{~B}$ & $2881.20 \mathrm{~B}$ & $9.83 \mathrm{AB}$ & $9.83 \mathrm{AB}$ & $3455.70 \mathrm{C}$ & $3464.1 \mathrm{E}$ & $1189.98 \mathrm{C}$ & $1177.70 \mathrm{EF}$ \\
\hline & $30 \mathrm{ppm}$ & $109.16 \mathrm{~B}$ & $123.60 \mathrm{~A}$ & $25.33 \mathrm{~A}$ & $27.33 \mathrm{~A}$ & $3298.00 \mathrm{~A}$ & $2947.96 \mathrm{~A}$ & $10.33 \mathrm{~A}$ & $10.33 \mathrm{~A}$ & $4337.93 \mathrm{~A}$ & $4431.8 \mathrm{~A}$ & $1415.85 \mathrm{~A}$ & $1407.23 \mathrm{~A}$ \\
\hline \multirow{2}{*}{ 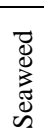 } & $1 \mathrm{~g} / \mathrm{L}$ & $107.83 \mathrm{BC}$ & 105.42DE & $19.83 \mathrm{BC}$ & 23.83BCD & $2718.25 \mathrm{~B}$ & $2690.43 \mathrm{D}$ & $9.33 \mathrm{ABC}$ & $9.33 \mathrm{ABC}$ & 3895.79 B & $3857.6 \mathrm{C}$ & $1393.33 \mathrm{AB}$ & $1297.35 \mathrm{D}$ \\
\hline & $2 \mathrm{~g} / \mathrm{L}$ & $119.00 \mathrm{~A}$ & $120.14 \mathrm{~B}$ & $24.50 \mathrm{AB}$ & $24.16 \mathrm{BC}$ & 2752.86 B & 2885.65 B & $9.83 \mathrm{AB}$ & $9.83 \mathrm{AB}$ & 4346.86 A & 4294.2B & $1361.70 \mathrm{AB}$ & 1407.23 A \\
\hline
\end{tabular}


Table 3: The Interaction average values of some vegetative growth of artichoke plants as affected by cultivars, NNA, GA 3,6BA and Seaweed Extract during the fall seasons of 201 6and 2017.

\begin{tabular}{|c|c|c|c|c|c|c|c|c|c|c|c|c|c|}
\hline \multicolumn{2}{|c|}{ Treatments } & \multicolumn{2}{|c|}{$\begin{array}{c}\text { Plant height /plant * } \\
(\mathbf{c m})\end{array}$} & \multicolumn{2}{|c|}{$\begin{array}{c}\text { Number of } \\
\text { leaves/plant }\end{array}$} & \multicolumn{2}{|c|}{$\begin{array}{c}\text { leave area /plant } \\
\left(\mathrm{cm}^{2}\right)\end{array}$} & \multicolumn{2}{|c|}{$\begin{array}{c}\text { Number of } \\
\text { branches/plant }\end{array}$} & \multicolumn{2}{|c|}{$\begin{array}{c}\text { Fresh weight /plant } \\
\text { (gm) }\end{array}$} & \multicolumn{2}{|c|}{$\begin{array}{c}\text { Dry weight /plant } \\
\text { (gm) }\end{array}$} \\
\hline & & $\mathbf{1}^{\text {st }_{\text {season }}}$ & $\mathbf{2}^{\text {nd }}{ }_{\text {season }}$ & $\mathbf{1}^{\text {st }}{ }_{\text {season }}$ & $2^{\text {nd }}{ }_{\text {season }}$ & $\mathbf{1}^{\text {st }}{ }_{\text {season }}$ & $2^{\text {nd }}{ }_{\text {season }}$ & $\mathbf{1}^{\text {st }}$ season & $\mathbf{2}^{\text {nd }}{ }_{\text {season }}$ & $\mathbf{1}^{\text {st }}$ season & $2^{\text {nd }}$ season & $\mathbf{1}^{\text {st }}$ season & $\mathbf{2}^{\text {nd }}{ }_{\text {season }}$ \\
\hline \multirow{9}{*}{$\begin{array}{l}\overline{0} \\
\overline{0} \\
\text { D. }\end{array}$} & Control & 102.66 efgh & $102.71 \mathrm{hi}$ & $20.33 \mathrm{bc}$ & $21.00 \mathrm{efg}$ & $1815.90 \mathrm{~g}$ & $1822.36 \mathrm{j}$ & $7.33 \mathrm{gh}$ & $6.33 \mathrm{~g}$ & $2132.67 \mathrm{k}$ & $3138.66 \mathrm{~m}$ & $591.11 \mathrm{i}$ & $589.97 \mathrm{j}$ \\
\hline & NAA $25 \mathrm{ppm}$ & 106.33 defg & 111.48def & $22.00 \mathrm{abc}$ & $24.33 \mathrm{bcd}$ & $3041.83 \mathrm{bc}$ & $3142.13 \mathrm{~b}$ & 8.67 efgh & $7.00 \mathrm{fg}$ & $3311.33 \mathrm{fgh}$ & $3229.71 \mathrm{j}$ & $1225.19 \mathrm{def}$ & $1186.36 \mathrm{f}$ \\
\hline & NAA50 ppm & $107.66 \mathrm{def}$ & $109.59 \mathrm{efg}$ & $22.33 \mathrm{abc}$ & $21.66 \mathrm{efg}$ & $3067.33 \mathrm{bc}$ & $2866.88 \mathrm{de}$ & 10.00 abcde & $9.66 \mathrm{bc}$ & 3548.33 ef & $3396.14 \mathrm{i}$ & $1244.52 \mathrm{def}$ & $1198.71 \mathrm{f}$ \\
\hline & $\mathrm{GA}_{3} 30 \mathrm{ppm}$ & $111.66 \mathrm{de}$ & $112.74 \mathrm{de}$ & $26.66 \mathrm{ab}$ & $23.00 \mathrm{cde}$ & $2976.06 \mathrm{bcd}$ & $2607.87 \mathrm{~g}$ & 10.33 abcde & $8.00 \mathrm{def}$ & $3399.00 \mathrm{fg}$ & $3379.34 \mathrm{i}$ & $1409.93 \mathrm{abc}$ & $1488.18 \mathrm{a}$ \\
\hline & $\mathrm{GA}_{3} 60 \mathrm{ppm}$ & $131.66 \mathrm{a}$ & $120.23 \mathrm{~b}$ & $21.67 \mathrm{abc}$ & $25.00 \mathrm{bc}$ & $2906.67 \mathrm{bcde}$ & $2961.14 \mathrm{c}$ & $10.67 \mathrm{abcd}$ & 8.66 bcde & $3801.00 \mathrm{de}$ & $3892.38 \mathrm{f}$ & $1249.53 \mathrm{def}$ & $1279.16 \mathrm{~d}$ \\
\hline & 6BA $20 \mathrm{ppm}$ & $121.66 \mathrm{bc}$ & $120.08 \mathrm{bc}$ & $26.33 \mathrm{ab}$ & $25.00 \mathrm{bc}$ & $2503.36 \mathrm{f}$ & $2858.87 \mathrm{e}$ & $11.00 \mathrm{abc}$ & $10.00 \mathrm{ab}$ & $3839.66 \mathrm{cde}$ & $3829.94 \mathrm{~g}$ & $1322.2 \mathrm{cde}$ & $1295.03 \mathrm{~d}$ \\
\hline & 6BA $30 \mathrm{ppm}$ & $130.33 \mathrm{ab}$ & $133.62 \mathrm{a}$ & $27.33 \mathrm{a}$ & $30.33 \mathrm{a}$ & $3604.36 \mathrm{a}$ & $3229.50 \mathrm{a}$ & $11.66 \mathrm{a}$ & $11.33 \mathrm{a}$ & $4542.33 \mathrm{a}$ & $4880.40 \mathrm{a}$ & $1506.30 \mathrm{a}$ & $1471.96 \mathrm{a}$ \\
\hline & Seaweed1g/L & $113.00 \mathrm{~cd}$ & 111.46def & $25.66 \mathrm{ab}$ & $24.00 \mathrm{bcd}$ & 2846.33 bcdef & $2896.91 \mathrm{e}$ & $11.33 \mathrm{ab}$ & $9.33 \mathrm{bcd}$ & $4211.66 \mathrm{~b}$ & $4218.91 \mathrm{c}$ & $1482.56 \mathrm{ab}$ & $1395.33 \mathrm{~b}$ \\
\hline & Seaweed $2 \mathrm{~g} / \mathrm{L}$ & $115.66 \mathrm{~cd}$ & $132.27 \mathrm{a}$ & $21.33 \mathrm{abc}$ & $25.33 \mathrm{~b}$ & $3183.17 \mathrm{~b}$ & $3154.82 \mathrm{~b}$ & $11.66 \mathrm{a}$ & $10.00 \mathrm{ab}$ & $4750.66 \mathrm{a}$ & $4450.58 \mathrm{~b}$ & $1488.2 \mathrm{a}$ & $1478.96 \mathrm{a}$ \\
\hline \multirow{9}{*}{$\begin{array}{l}\frac{\overrightarrow{0}}{\tilde{T}} \\
\frac{\pi}{\pi} \\
0\end{array}$} & Control & $96.00 \mathrm{~h}$ & $94.77 \mathrm{j}$ & $17.67 \mathrm{c}$ & $20.66 \mathrm{fg}$ & $1616.15 \mathrm{~g}$ & $1620.30 \mathrm{k}$ & $7.00 \mathrm{~h}$ & 8.33 cdef & $1898.07 \mathrm{k}$ & $1897.66 \mathrm{n}$ & $437.09 \mathrm{j}$ & $450.72 \mathrm{k}$ \\
\hline & NAA $25 \mathrm{ppm}$ & $97.66 \mathrm{gh}$ & $100.20 \mathrm{i}$ & $18.00 \mathrm{c}$ & $20.00 \mathrm{~g}$ & 2676.81cdef & $2620.28 \mathrm{~g}$ & $8.00 \mathrm{fgh}$ & 7.66 efg & $2913.97 \mathrm{ij}$ & $2860.27 \mathrm{I}$ & $1078.17 \mathrm{gh}$ & $1090.65 \mathrm{~h}$ \\
\hline & NAA $50 \mathrm{ppm}$ & $95.66 \mathrm{~h}$ & $109.10 \mathrm{efg}$ & $20.33 \mathrm{bc}$ & $23.00 \mathrm{cde}$ & 2529.65 ef & $2509.00 \mathrm{~h}$ & 9.00 defg & $8.00 \mathrm{def}$ & $3193.50 \mathrm{ghi}$ & $3197 . .53 \mathrm{j}$ & $1120.07 \mathrm{fgh}$ & $1136.50 \mathrm{~g}$ \\
\hline & $\mathrm{GA}_{3} 30 \mathrm{ppm}$ & $100.00 \mathrm{fgh}$ & $115.55 \mathrm{~cd}$ & $23.33 \mathrm{bc}$ & $24.33 \mathrm{bcd}$ & $2705.69 \mathrm{cdef}$ & $2698.26 \mathrm{f}$ & 9.33 cdef & $9.33 \mathrm{bcd}$ & $2889.15 \mathrm{j}$ & $2905.53 \mathrm{I}$ & $1235.206 \mathrm{def}$ & $1274.82 \mathrm{~d}$ \\
\hline & $\mathrm{GA}_{3} 60 \mathrm{ppm}$ & $102.66 \mathrm{efgh}$ & $113.58 \mathrm{de}$ & $18.67 \mathrm{c}$ & $24.00 \mathrm{bcd}$ & 2528.80 ef & $2503.26 \mathrm{~h}$ & 9.67 bcdef & $9.00 \mathrm{bcde}$ & $3306.87 \mathrm{fgh}$ & $3509.36 \mathrm{~h}$ & $1087.09 \mathrm{gh}$ & $1089.43 \mathrm{~h}$ \\
\hline & 6BA $20 \mathrm{ppm}$ & $97.33 \mathrm{gh}$ & 110.59 ef & $18.33 \mathrm{c}$ & $21.00 \mathrm{efg}$ & $2002.69 \mathrm{~g}$ & $2009.10 \mathrm{i}$ & 9.33 cdef & $9.66 \mathrm{bc}$ & 3071.73hij & $3098.33 \mathrm{k}$ & $1057.76 \mathrm{~h}$ & $1060.38 \mathrm{i}$ \\
\hline & 6BA $30 \mathrm{ppm}$ & $111.33 \mathrm{de}$ & $122.02 \mathrm{~b}$ & $21.33 \mathrm{abc}$ & $24.33 \mathrm{bcd}$ & 2991.62 bc & $2904.41 \mathrm{~d}$ & 10.33 abcde & $10.00 \mathrm{ab}$ & $4133.52 \mathrm{bc}$ & $4137.81 \mathrm{~d}$ & $1349.13 \mathrm{bc}$ & $1342.50 \mathrm{c}$ \\
\hline & Seaweed1g/L & $102.66 \mathrm{efgh}$ & $105.39 \mathrm{gh}$ & $23.33 \mathrm{abc}$ & $20.66 \mathrm{fg}$ & 2590.16 def & $2483.77 \mathrm{~h}$ & $10.66 \mathrm{abcd}$ & $9.33 \mathrm{bcd}$ & 3579.91 ef & $3496.43 \mathrm{~h}$ & 1198.44 efg & $1199.41 \mathrm{f}$ \\
\hline & Seaweed $2 \mathrm{~g} / \mathrm{L}$ & $107.66 \mathrm{def}$ & $108.01 \mathrm{fg}$ & $22.33 \mathrm{abc}$ & $22.66 \mathrm{def}$ & 2760.60 cdef & $2741.09 \mathrm{f}$ & 10.00 abcde & 8.66 bcde & $3943.05 \mathrm{bcd}$ & $3983.30 \mathrm{e}$ & $1280.35 \mathrm{cde}$ & $1226.40 \mathrm{e}$ \\
\hline
\end{tabular}


N-6-benzyle amino purine and seaweed extract with all concentrations showed significant effects on plant height, number of leaves, leaf area, number of branches, fresh and dry weight compared to the control treatment, in both seasons. It is obvious that N-6-benzyle amino purine at $30 \mathrm{ppm}$ gave the highest mean values of all studied characters, followed by gibberellic acid at $60 \mathrm{ppm}$, seaweed extract at $2 \mathrm{~g} / \mathrm{l}$ and naphthalene acetic acid at 50 ppm. However, plant height reached the maximum when plants sprayed with seaweed at $2 \mathrm{~g} / \mathrm{l}$, in the first season. This particular treatment (N-6-benzyle amino purine at $30 \mathrm{ppm}$ ), the estimated percentages increase in plant height, number of leaves, leaf area, number of branches, fresh and dry weight were (9.89 and $25.17 \%)$, (33.31 and 31.20\%), (92.19 and $71.26 \%)$, (55.79 and $40.92 \%)$, (115.24 and $119.59 \%)$, and (175.40 and $170.43 \%)$, compared to the control treatment in the first and second season, respectively. The present results could be attributed to the role of each spraying material. Synthetic cytokinin such as benzyl amino purine (BAP), 2chloro-4-pyridyl-Nphenylurea (CPPU) and 6furfuryl-aminopurin (Kinetin) can improve plant growth by cell division, break bud dormancy and promotes the growth of the lateral bud (Hossain et al., 2006). Exogenous application of N-6-benzyle amino purine on Cowpea improved vegetative growth and that seemed to be due to increase greater chlorophyll synthesis as observed by Reddy et al., (2009). Application of the 6BA on okra plants increased significantly plant height, number of leaves, number of branches and leaf area plant ${ }^{-1}$ compared to the untreated control (Brengi ,2018). Using benzyl adenine at $100-200 \mathrm{ppm}$ led to significant increases in branch, dry weight, period from showing color to full opening stage, flowering duration and inflorescence (Gabrelel et al., 2018). Cytokinin reduced heat stress effects and increased production of artichoke growing under hot fall conditions (Schrader, 2005). The endogenous cytokinin levels decreased under high temperature conditions (Hare et al., 1997; Banowetz et al., 1999).

The interaction effects between artichoke cultivars and the spraying treatments on vegetative growth parameters of artichoke plants, were significance during both seasons (Table, 3). The combined treatment of France cultivar and N-6benzyle amino purine at $30 \mathrm{ppm}$, generally, recorded the highest mean values of plant height, number of leaves, leaf area, number of branches, fresh and dry weight compared to the control treatment, in both seasons.

\section{Yield and its components:}

Tables (4 and 5) show the main effects of the two studied factors of artichoke cultivars and the spraying treatments (gibberellic acid, naphthalene acetic acid, N-6-benzyle amino purine and seaweed extract) and their interactions on the yield and its components of artichoke plants in 2016-2017 and 2017-2018 seasons.

Results outlined in Table (4) exhibit the average values of number of early heads/plant, number of early heads/feddan, total number of heads/ plant, and weight of edible part of artichoke plants in 2016-2017 and 2017-2018 seasons. The results indicated that the main effect of artichoke cultivars differed significantly in their yield parameters in both seasons. Balady cultivar showed higher mean values of yield and its component than France cultivar, in both seasons. The noticed differences between the two cultivars could be attributed to their genetic features. This finding is in agreement with those of McErlich (1983), who compared three cultivar of globe artichoke in a three years' trial. He found that Tudella and E15 gave higher yield than Green Globe cv. which gave the highest average head weight. In another study, Rodrigo et al. (1979) evaluated five introduced artichoke cultivars in Spain (Murcia, Amposta, Tudela, San Juan de Enova and Tudela X Amposta). Early yield was about $15-25 \%$ of the total yield of artichoke harvested during November to early March.

Calabrese et al. (1994) showed significant difference among some tested cultivar in their number of heads and earliness. They added that 21 heads $/ \mathrm{m}^{2}$ were harvested 19 times between November and May. The greatest number of heads was 209000 heads /ha (on average) compared with only 143000 heads/ha for cultivars 137. Moreover, Basnitzki and Zohary (1987) revealed that Talpiot artichoke cultivar gives yield of 13-16 ton of fresh heads/hectare. Such results have been also obtained with colonel varieties Violet de Provence and Blanc de Hyeres cultivars. Pandita et al. (1988) evaluated exotic cultivars for yield characters of globe artichoke in Kashmir valley. They found that green globe (GG) and Fl Sasanqua gave the highest yields based on number of buds/plant (20.2 and 20.0, respectively) and bud weight $\mathrm{kg} /$ plant (6.1 and 5.3, respectively).

Miccolis et al. (1990) found that the peak yield of artichoke was seen from April to mid-May. The average harvesting period was 50 days, but it varied from only 7 days in Violet to Spinoso to 201 days in Blanco cultivar. They added that average yield/plant was $843.5 \mathrm{~g}$ and ranged from $119 \mathrm{~g}$ to $1832 \mathrm{~g}$. Recently, Soria et al. (2020) showed that the greatest final total yield was reached by the green head cultivars/lines 'Nun 4011' (3.73 $\left.\mathrm{kg} \mathrm{m}^{-2}\right)$ and 'Madrigal' (3.65 $\mathrm{kg} \mathrm{m}^{-2}$ ) and among purple color head cultivar 'Opal' $\left(2.92 \mathrm{~kg} \mathrm{~m}^{-2}\right)$. Similar results were obtained with those of Gabr and Sarg (1998) and Gabr et al. (2002) on potatoes. 
Table 4: The main average values of Yield and its components of artichoke plants as affected by cultivars, NAA, GA 6 6BA and Seaweed Extract during the fall season of 2016 - 2017 and 2017-2018.

\begin{tabular}{|c|c|c|c|c|c|c|c|c|c|c|c|}
\hline \multirow{3}{*}{\multicolumn{2}{|c|}{ Treatments }} & \multicolumn{5}{|c|}{ Early yield } & \multicolumn{5}{|c|}{ Total yield } \\
\hline & & \multicolumn{2}{|c|}{ Number of heads /plant * } & \multicolumn{2}{|c|}{ Number of heads / feddan } & \multicolumn{2}{|c|}{$\begin{array}{l}\text { Total number of } \\
\text { heads / plant }\end{array}$} & \multicolumn{2}{|c|}{$\begin{array}{l}\text { Total number of heads } \\
\text { /feddan }\end{array}$} & \multicolumn{2}{|c|}{$\begin{array}{l}\text { Wight of edible part } \\
\text { /plant (gm). }\end{array}$} \\
\hline & & $1^{\text {st }}$ season & $2^{\text {nd }}$ season & $1^{\text {st }}$ season & $2^{\text {nd }}$ season & $1^{\text {st }}$ season & $2^{\text {nd }}$ season & $1^{\text {st }}$ season & $2^{\text {nd }}$ season & $1^{\text {st }}$ season & $2^{\text {nd }}$ season \\
\hline French & & $5.09 \mathrm{~B}$ & $5.36 \mathrm{~B}$ & $27145 \mathrm{~B}$ & $28585 \mathrm{~B}$ & $11.75 \mathrm{~B}$ & $11.36 \mathrm{~B}$ & $62653.86 \mathrm{~B}$ & $60574.47 \mathrm{~B}$ & $56.90 \mathrm{~B}$ & $61.76 \mathrm{~B}$ \\
\hline \multirow[t]{10}{*}{ Balady } & & $6.06 \mathrm{~A}$ & $5.78 \mathrm{~A}$ & $32318 \mathrm{~A}$ & $30825 \mathrm{~A}$ & $12.89 \mathrm{~A}$ & $12.32 \mathrm{~A}$ & $68729.04 \mathrm{~A}$ & $65702.97 \mathrm{~A}$ & $63.93 \mathrm{~A}$ & $62.28 \mathrm{~A}$ \\
\hline & Control(tap water) & $4.30 \mathrm{D}$ & $4.74 \mathrm{E}$ & $22932 \mathrm{D}$ & $25278 \mathrm{E}$ & $9.87 \mathrm{G}$ & $11.12 \mathrm{~F}$ & $52656.71 \mathrm{G}$ & $59295.49 \mathrm{~F}$ & $59.93 \mathrm{CDE}$ & $61.95 \mathrm{CD}$ \\
\hline & NAA 25 ppm & $5.01 \mathrm{C}$ & $5.02 \mathrm{DE}$ & $26718 \mathrm{C}$ & $26772 \mathrm{DE}$ & $11.25 \mathrm{~F}$ & $10.65 \mathrm{G}$ & $59996.25 \mathrm{~F}$ & $56777.52 \mathrm{G}$ & $58.63 \mathrm{E}$ & $57.32 \mathrm{E}$ \\
\hline & NAA $50 \mathrm{ppm}$ & $5.13 \mathrm{C}$ & $5.07 \mathrm{DE}$ & $27358 \mathrm{C}$ & $27038 \mathrm{DE}$ & $11.62 \mathrm{E}$ & $11.25 \mathrm{E}$ & $61976.13 \mathrm{E}$ & $60014.92 \mathrm{E}$ & $60.92 \mathrm{BCD}$ & $61.74 \mathrm{CD}$ \\
\hline & $\mathrm{GA}_{3} 30 \mathrm{ppm}$ & $5.15 \mathrm{C}$ & $5.51 \mathrm{BCD}$ & $27465 \mathrm{C}$ & 29385 BCD & $11.50 \mathrm{E}$ & $11.36 \mathrm{E}$ & $61336.17 \mathrm{E}$ & $60582.88 \mathrm{E}$ & $59.68 \mathrm{DE}$ & $61.78 \mathrm{CD}$ \\
\hline & $\mathrm{GA}_{3} 60 \mathrm{ppm}$ & $6.16 \mathrm{~A}$ & $6.73 \mathrm{~A}$ & $32851 \mathrm{~A}$ & $35891 \mathrm{~A}$ & $12.50 \mathrm{D}$ & $12.32 \mathrm{C}$ & $66655.83 \mathrm{D}$ & $65694.56 \mathrm{C}$ & $61.33 \mathrm{BC}$ & $62.50 \mathrm{BC}$ \\
\hline & 6BA $20 \mathrm{ppm}$ & $5.24 \mathrm{C}$ & $5.21 \mathrm{CDE}$ & $27945 \mathrm{C}$ & $27785 \mathrm{CDE}$ & $12.87 \mathrm{C}$ & $12.23 \mathrm{C}$ & $68655.71 \mathrm{C}$ & $65240.19 \mathrm{C}$ & $58.50 \mathrm{E}$ & $62.14 \mathrm{CD}$ \\
\hline & 6BA $30 \mathrm{ppm}$ & $5.68 \mathrm{~B}$ & $6.03 \mathrm{~B}$ & $30291 \mathrm{~B}$ & $32158 \mathrm{~B}$ & $14.00 \mathrm{~B}$ & $12.66 \mathrm{~B}$ & $74655.33 \mathrm{~B}$ & $67512.05 \mathrm{~B}$ & $63.51 \mathrm{~A}$ & $65.90 \mathrm{~A}$ \\
\hline & Seaweed $1 \mathrm{~g} / \mathrm{L}$ & $5.87 \mathrm{~B}$ & $5.82 \mathrm{BC}$ & $31305 \mathrm{~B}$ & $31038 \mathrm{BC}$ & $12.75 \mathrm{CD}$ & $11.57 \mathrm{D}$ & $67975.75 \mathrm{CD}$ & $61708.68 \mathrm{D}$ & $58.97 \mathrm{E}$ & $60.70 \mathrm{D}$ \\
\hline & Seaweed $2 \mathrm{~g} / \mathrm{L}$ & $6.38 \mathrm{~A}$ & $6.02 \mathrm{~B}$ & $34025 \mathrm{~A}$ & $32105 \mathrm{~B}$ & $14.50 \mathrm{~A}$ & $13.39 \mathrm{~A}$ & $77315.17 \mathrm{~A}$ & $71422.2 \mathrm{~A}$ & $62.25 \mathrm{AB}$ & $64.15 \mathrm{AB}$ \\
\hline
\end{tabular}

* values having the same alphabetical letter (s) in common, do not significant different, using the revised L.S.D. test at 0.05 level of probability. 
Concerning the main effects of the spraying treatments (gibberellic acid, naphthalene acetic acid, N-6-benzyle amino purine and seaweed extract) on yield parameters of artichoke plants, are presented in Tables (4). Spraying the aforementioned treatments showed significant effects of early yield (number of early heads / plant and number of early heads /feddan) and total yield (number of heads/ plant, number of heads /feddan and weight of edible part plant), compared to control treatment, in both seasons. Regarding early yield, it is clear that either gibberellic acid at $60 \mathrm{ppm}$ in both seasons or seaweed extract at $2 \mathrm{~g} / \mathrm{l}$ in the first season only, gave the highest mean values of number of head/plant and number of head /feddan compared to other treatments. The gibberellic acid at $60 \mathrm{ppm}$ increased number of early head / plant and number of early head /feddan by 43.25 and $41.98 \%$, respectively, compared to the control treatment) as an average of the two seasons. These results are in harmony with those of George et al. (2008) who reported that increasing number of globe artichoke heads/plant, with spraying $\mathrm{GA}_{3}$ concentrations could be attributed to its mode of action in promotion of flowers primordia production. It is known that number of heads per plant is a vital factor, whereas this trait is directly in connection with the number of heads per feddan, number of early yield per plant and per feddan. It is concluded that gibberellic acid plays an important role in boosting the early yield. The earliness of globe artichoke plant yield may be taken place owing to $\mathrm{GA}_{3}$ treatments which promote vegetative growth and thus there is a translocation of the synthesized assimilates to other plant parts and might have facilitate early flowering (produced heads). Conspicuously, most globe artichoke growers inclined to foliar application of $\mathrm{GA}_{3}$ as a vernalized tool to enhance and accelerate heads initiation (early yield) and achieve high financial returns because of high prices. In Egypt, the highest market prices are recorded from December to February. Nonetheless, for earlier planting, globe artichoke floral induction requires such add $\mathrm{GA}_{3}$ treatment (250 hours at temperature $\left.\leq 7^{\circ} \mathrm{C}\right)$. Therefore, globe artichoke growers turn to use $\mathrm{GA}_{3}$ to overcame the higher temperature during the early time-course of plantation. The results of the present study are in agreement with those of EL-Baz et al. (1979), who stated that foliar application of $\mathrm{GA}_{3}$ at either 50 or $75 \mathrm{ppm}$, induced early yield in selected "French" cv., but did not exhibit such influence on the total yield. Also, Basnitzki and Zohary (1987) showed that foliar application of globe artichoke plants with $\mathrm{GA}_{3}$ at 60 and $120 \mathrm{ppm}$ can be used safely. Zaki et al. (1991) reported that foliar application of $\mathrm{GA}_{3}$ at 50,100 and $200 \mathrm{ppm}$ on globe artichoke plants cv. 'Herious'; improved early and total yield of flower head, especially at $200 \mathrm{ppm}$. Likewise, Lin et al. (1991) in Taiwan, reported that foliar application of $\mathrm{GA}_{3}$ at $45 \mathrm{ppm}$ in February and March, enhanced positive effect on bolting earliness, bolting percentage and flower bud yield. Also, Schrader and keith (1997) found that application of 20 ppm $\mathrm{GA}_{3}$ three times at 2 weeks' intervals increased significantly the percentage of early yield. Parallel reports about the effect of $\mathrm{GA}_{3}$ on encouraging earliness of globe artichoke were, also, introduced by El-Gridly (1994), Schrader (1994) and Miguel et al. (2003).

The positive effect of seaweeds extract on early yield characters was reported by Saif- Eldeen et al. (2014) who showed that the high level of seaweeds extract were accompanied with significant yield distribution (early, medium and late yields) compared with control.

Concerning total yield, It is obvious that spraying seaweed extract at $2 \mathrm{~g} / \mathrm{l}$ gave the highest mean values of number of total heads/plant and number of total head s/feddan, in both seasons, compared to the other treatments. However, the highest mean values of the weight of edible part/ head were achieved when plants sprayed with N-6benzyle amino purine at $30 \mathrm{ppm}$, in both seasons, compared to the other treatments. At the favorite treatment (seaweed extract at $2 \mathrm{~g} / \mathrm{l}$ ), the estimated percentages increase in number of total heads/plant and number of total heads /feddan were (46.90 and $20.41 \%$ ) and (46.82 and $50.45 \%$ ), respectively for the first and second seasons, respectively, compared to the control treatment. The positive effects of seaweed extract in yield parameters could be due to the fact that it contains macro, micronutrients and amino acids that improve nutritional status, vegetative growth and yield. These results are in harmony with Saif- Eldeen et al. (2014) who showed that the increasing seaweeds extract levels were accompanied with significant yield distribution (early, medium and late yields) compared with control. Also, Seaweed extract application for different crops has a great importance due to its content of organic matter, micro elements ( $\mathrm{Fe}, \mathrm{Cu}$, $\mathrm{Zn}, \mathrm{Co}, \mathrm{Mo}, \mathrm{Mn}$ and $\mathrm{Ni}$ ), vitamins and amino acids. Additionally, it is also rich in growth regulators such as auxins, cytokinins and gibberellins (Khan et al., 2009; Hamed 2012; El- Miniawy et al.2014). The interaction effects between artichoke cultivars and the spraying treatments on yield parameters of artichoke plants were significant during both seasons (Table 5). The combined treatment of Balady cultivar and either gibberellic acid at $60 \mathrm{ppm}$ or seaweed extract at $2 \mathrm{~g} / \mathrm{L}$, generally, recorded the highest mean values of number of early yield (number of early heads/plant and number of early heads /feddan) compared to the other treatments, in both seasons. 
Table 5: The interaction average values of yield and its components of artichoke plants as affected by cultivars, NAA, GA3 6BA and Seaweed Extract during fall season of 2016-2017 and2017-2018.

\begin{tabular}{|c|c|c|c|c|c|c|c|c|c|c|c|}
\hline & \multirow{3}{*}{ Treatments } & \multicolumn{4}{|c|}{ Early yield } & \multicolumn{6}{|c|}{ Total yield } \\
\hline & & \multicolumn{2}{|c|}{$\begin{array}{c}\text { Number of *heads / } \\
\text { plant }\end{array}$} & \multicolumn{2}{|c|}{$\begin{array}{c}\text { Number of heads / } \\
\text { feddan }\end{array}$} & \multicolumn{2}{|c|}{$\begin{array}{c}\text { Number of heads / } \\
\text { plant }\end{array}$} & \multicolumn{2}{|c|}{$\begin{array}{c}\text { Number of heads / } \\
\text { feddan }\end{array}$} & \multicolumn{2}{|c|}{$\begin{array}{c}\text { Wight of edible part } \\
\text { /plant (gm) }\end{array}$} \\
\hline & & $1^{\text {st }}$ season & $2^{\text {nd }}$ season & $1^{\text {st }}$ season & $2^{\text {nd }}$ season & $1^{\text {st }}$ season & $2^{\text {nd }}$ season & $1^{\text {st }}$ season & $2^{\text {nd }}$ season & $1^{\text {st }}$ season & $2^{\text {nd }}$ season \\
\hline \multirow{9}{*}{ 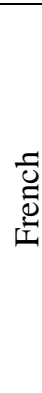 } & Control(tap water) & $3.93 \mathrm{i}$ & $4.43 \mathrm{~h}$ & $20959 \mathrm{i}$ & $23625 \mathrm{~h}$ & $9.00 \mathrm{q}$ & $10.41 \mathrm{j}$ & $47997.00 \mathrm{q}$ & $55516.53 \mathrm{j}$ & $812.6 \mathrm{i}$ & 810.991 \\
\hline & NAA $25 \mathrm{ppm}$ & $4.75 \mathrm{~h}$ & $4.90 \mathrm{fgh}$ & $25332 \mathrm{~h}$ & 26132 fgh & $10.50 \mathrm{p}$ & $10.17 \mathrm{k}$ & $55996.50 \mathrm{p}$ & $54236.61 \mathrm{k}$ & $759.49 \mathrm{i}$ & $917.12 \mathrm{j}$ \\
\hline & NAA $50 \mathrm{ppm}$ & $4.73 \mathrm{~h}$ & $4.75 \mathrm{gh}$ & $25225 \mathrm{~h}$ & $25332 \mathrm{gh}$ & $11.00 \mathrm{n}$ & $10.65 \mathrm{ij}$ & $58636.33 \mathrm{n}$ & $56796.45 \mathrm{ij}$ & $887.55 \mathrm{~h}$ & $853.95 \mathrm{k}$ \\
\hline & $\mathrm{GA}_{3} 30 \mathrm{ppm}$ & $4.61 \mathrm{~h}$ & 5.21 defgh & $24585 \mathrm{~h}$ & 27785 defgh & $11.25 \mathrm{~m}$ & $11.13 \mathrm{gh}$ & $59996.25 \mathrm{~m}$ & $59356.29 \mathrm{gh}$ & $807.3 \mathrm{i}$ & $913.35 \mathrm{j}$ \\
\hline & $\mathrm{GA}_{3} 60 \mathrm{ppm}$ & $4.70 \mathrm{~h}$ & $4.85 \mathrm{gh}$ & $25065 \mathrm{~h}$ & $25865 \mathrm{gh}$ & 11.751 & $12.07 \mathrm{e}$ & 62636.081 & $64369.31 \mathrm{e}$ & 986 efg & $1045.84 \mathrm{de}$ \\
\hline & 6BA $20 \mathrm{ppm}$ & $5.11 \mathrm{~g}$ & 5.66 bcdefg & $27252 \mathrm{~g}$ & 30185 bcdefg & $12.75 \mathrm{~h}$ & $12.54 \mathrm{~d}$ & $67995.75 \mathrm{~h}$ & $66875.82 \mathrm{~d}$ & $956.8 \mathrm{~g}$ & $1027.77 \mathrm{ef}$ \\
\hline & 6BA $30 \mathrm{ppm}$ & $5.60 \mathrm{ef}$ & 5.58 bcdefg & 29865 ef & 29758bcdefg & $13.50 \mathrm{e}$ & $11.36 \mathrm{fg}$ & $71995.50 \mathrm{e}$ & $60582.88 \mathrm{fg}$ & $977.13 \mathrm{fg}$ & $1129.82 c$ \\
\hline & Seaweed $1 \mathrm{~g} / \mathrm{L}$ & $5.17 \mathrm{~g}$ & 5.24 cdefgh & $27572 \mathrm{~g}$ & 27945 cdefgh & 11.751 & $13.25 \mathrm{~b}$ & 62636.081 & $56796.45 \mathrm{ij}$ & $812.6 \mathrm{i}$ & $982.94 \mathrm{~g}$ \\
\hline & Seaweed $2 \mathrm{~g} / \mathrm{L}$ & $6.06 \mathrm{~cd}$ & 5.93 bcde & $32318 \mathrm{~cd}$ & 31625 bccde & & & $75995.25 \mathrm{c}$ & $70662.25 \mathrm{~b}$ & $1087.32 \mathrm{~d}$ & $1123.87 \mathrm{c}$ \\
\hline \multirow{9}{*}{ 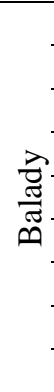 } & Control tap water & $4.67 \mathrm{~h}$ & 5.05 efgh & $24905 \mathrm{~h}$ & 26932 efgh & $10.75 \mathrm{o}$ & $11.83 \mathrm{e}$ & $57316.42 \mathrm{o}$ & $63249.38 \mathrm{e}$ & $1073.57 \mathrm{~d}$ & $962.95 \mathrm{~h}$ \\
\hline & NAA 25 ppm & $5.27 \mathrm{fg}$ & 5.14 efgh & $28105 \mathrm{fg}$ & 27412efgh & & & $63996.00 \mathrm{k}$ & $59302.96 \mathrm{~h}$ & $1006.47 \mathrm{efg}$ & $955.26 \mathrm{~h}$ \\
\hline & NAA $50 \mathrm{ppm}$ & $5.53 \mathrm{ef}$ & 5.38 cdefg & $29491 \mathrm{ef}$ & 28692 cdefg & $12.25 \mathrm{j}$ & $11.86 \mathrm{e}$ & $65315.92 \mathrm{j}$ & $63622.69 \mathrm{e}$ & $1079.82 \mathrm{~d}$ & $1048.09 \mathrm{~d}$ \\
\hline & $\mathrm{GA}_{3} 30 \mathrm{ppm}$ & $5.69 \mathrm{e}$ & $5.81 \mathrm{bcdef}$ & $30345 \mathrm{e}$ & 30985bcdef & 11.751 & $11.59 \mathrm{f}$ & 62676.081 & $61809.47 \mathrm{f}$ & 1021.44 ef & $1023.24 \mathrm{f}$ \\
\hline & $\mathrm{GA}_{3} 60 \mathrm{ppm}$ & $6.72 \mathrm{a}$ & $7.30 \mathrm{a}$ & $35838 \mathrm{a}$ & $38931 \mathrm{a}$ & $13.25 \mathrm{f}$ & $12.57 \mathrm{~d}$ & $70675.58 \mathrm{f}$ & $67035.81 \mathrm{~d}$ & $1156.34 \mathrm{c}$ & $1050.14 \mathrm{~d}$ \\
\hline & 6BA $20 \mathrm{ppm}$ & $5.78 \mathrm{de}$ & $6.17 \mathrm{bc}$ & $30825 \mathrm{de}$ & $32905 b c$ & $13.00 \mathrm{~g}$ & $11.93 \mathrm{e}$ & $69315.67 \mathrm{~g}$ & $63089.39 \mathrm{e}$ & $1035.89 \mathrm{de}$ & $1052.69 \mathrm{~d}$ \\
\hline & 6BA $30 \mathrm{ppm}$ & $6.25 \mathrm{bc}$ & $6.41 \mathrm{ab}$ & $33331 \mathrm{bc}$ & $34185 \mathrm{ab}$ & $14.50 \mathrm{~b}$ & $13.96 \mathrm{a}$ & $77315.17 \mathrm{~b}$ & $74448.68 \mathrm{a}$ & $1321.55 \mathrm{a}$ & $1308.37 \mathrm{a}$ \\
\hline & Seaweed $1 \mathrm{~g} / \mathrm{L}$ & $6.55 \mathrm{ab}$ & $6.13 \mathrm{bcd}$ & $34931 \mathrm{ab}$ & $32691 \mathrm{bcd}$ & $13.75 \mathrm{~d}$ & $12.89 \mathrm{c}$ & $73315.42 \mathrm{~d}$ & $68742.37 \mathrm{c}$ & $1083.43 \mathrm{~d}$ & $1133.37 \mathrm{c}$ \\
\hline & Seaweed 2g/L & $6.69 \mathrm{a}$ & $6.38 \mathrm{ab}$ & $35678 \mathrm{a}$ & $34025 \mathrm{ab}$ & $14.75 \mathrm{a}$ & $13.13 \mathrm{bc}$ & $78635.08 \mathrm{a}$ & $70022.29 \mathrm{bc}$ & $1212.68 \mathrm{~b}$ & $1208.61 \mathrm{~b}$ \\
\hline
\end{tabular}

* values having the same alphabetical letter (s) in common, do not significant different, using the revised L.S.D. test at 0.05 level of probability. 
Table 6: The main average values of some chemical analysis of artichoke leaf as affected by cultivars, NAA, GA3 ,6BA and Seaweed Extract during the fall Season of 2016-2017 and 2017- 2018.

\begin{tabular}{|c|c|c|c|c|c|c|c|c|c|c|c|}
\hline \multirow[t]{2}{*}{ Treatments } & & \multicolumn{2}{|c|}{ Total chlorophyll * } & \multicolumn{2}{|c|}{$\mathbf{N}(\%)$} & \multicolumn{2}{|c|}{$\mathbf{P}(\%)$} & \multicolumn{2}{|c|}{$\mathrm{K}(\%)$} & \multicolumn{2}{|c|}{ Protein $(\%)$} \\
\hline & & $1^{\text {st }}$ season & $2^{\text {nd }}$ season & $1^{\text {st }}$ season & $2^{\text {nd }}$ season & $1^{\text {st }}$ season & $2^{\text {nd }}$ season & $1^{\text {st }}$ season & $2^{\text {nd }}$ season & $1^{\text {st }}$ season & $2^{\text {nd }}$ season \\
\hline \multirow{2}{*}{ treatments } & French & $46.75 \mathrm{~A}$ & $45.94 \mathrm{~A}$ & $2.91 \mathrm{~A}$ & $2.84 \mathrm{~A}$ & $0.71 \mathrm{~A}$ & $0.72 \mathrm{~A}$ & $3.40 \mathrm{~B}$ & $3.40 \mathrm{~B}$ & $18.19 \mathrm{~A}$ & $17.77 \mathrm{~A}$ \\
\hline & Balady & $46.75 \mathrm{~A}$ & $46.38 \mathrm{~A}$ & $2.88 \mathrm{~B}$ & $2.86 \mathrm{~A}$ & $0.74 \mathrm{~A}$ & $0.76 \mathrm{~A}$ & $3.58 \mathrm{~A}$ & $3.64 \mathrm{~A}$ & $17.97 \mathrm{~B}$ & $17.85 \mathrm{~A}$ \\
\hline Control & tap water & $44.11 \mathrm{D}$ & $47.47 \mathrm{E}$ & $2.56 \mathrm{C}$ & $2.48 \mathrm{~F}$ & $0.64 \mathrm{~F}$ & $0.65 \mathrm{E}$ & $3.27 \mathrm{E}$ & $3.26 \mathrm{DE}$ & $15.97 \mathrm{C}$ & $15.48 \mathrm{~F}$ \\
\hline \multirow{2}{*}{ NAA } & $25 \mathrm{ppm}$ & $44.40 \mathrm{C}$ & $44.67 \mathrm{DE}$ & $2.78 \mathrm{~B}$ & $2.70 \mathrm{E}$ & $0.71 \mathrm{BCD}$ & $0.71 \mathrm{D}$ & $3.37 \mathrm{D}$ & $3.32 \mathrm{CDE}$ & $17.40 \mathrm{~B}$ & $16.90 \mathrm{E}$ \\
\hline & $50 \mathrm{ppm}$ & $47.35 \mathrm{~A}$ & $46.44 \mathrm{ABC}$ & $2.81 \mathrm{~B}$ & $2.93 \mathrm{BC}$ & $0.73 \mathrm{SB}$ & $0.75 \mathrm{ABCD}$ & $3.36 \mathrm{D}$ & $3.17 \mathrm{E}$ & $17.57 \mathrm{~B}$ & $18.32 \mathrm{BC}$ \\
\hline \multirow{2}{*}{$\mathrm{GA}_{3}$} & $30 \mathrm{ppm}$ & $47.27 \mathrm{~A}$ & $45.92 \mathrm{BC}$ & $2.92 \mathrm{~A}$ & $2.96 \mathrm{AB}$ & $0.70 \mathrm{DE}$ & $0.76 \mathrm{ABC}$ & $3.42 \mathrm{D}$ & $3.43 \mathrm{BCD}$ & $18.26 \mathrm{~A}$ & $18.48 \mathrm{AB}$ \\
\hline & $60 \mathrm{ppm}$ & $46.35 \mathrm{AB}$ & $46.85 \mathrm{AB}$ & $2.92 \mathrm{~A}$ & $2.89 \mathrm{BC}$ & $0.70 \mathrm{CDE}$ & $0.73 \mathrm{BCD}$ & $3.53 \mathrm{BC}$ & $3.63 \mathrm{AB}$ & $18.27 \mathrm{~A}$ & $18.07 \mathrm{BC}$ \\
\hline \multirow{2}{*}{$6 \mathrm{BA}$} & $20 \mathrm{ppm}$ & $47.23 \mathrm{~A}$ & $45.57 \mathrm{CD}$ & $2.85 \mathrm{~B}$ & $2.79 \mathrm{D}$ & $0.73 \mathrm{AB}$ & $0.72 \mathrm{CD}$ & $3.50 \mathrm{C}$ & $3.68 \mathrm{~A}$ & $17.79 \mathrm{~B}$ & $17.46 \mathrm{D}$ \\
\hline & $30 \mathrm{ppm}$ & $46.28 \mathrm{AB}$ & $47.01 \mathrm{~A}$ & $2.94 \mathrm{~A}$ & $2.95 \mathrm{AB}$ & $0.75 \mathrm{~A}$ & $0.79 \mathrm{~A}$ & $3.52 \mathrm{BC}$ & $3.72 \mathrm{~A}$ & $18.36 \mathrm{~A}$ & $18.46 \mathrm{AB}$ \\
\hline \multirow{2}{*}{ Seaweed } & $1 \mathrm{~g} / \mathrm{L}$ & $47.10 \mathrm{AB}$ & $46.85 \mathrm{AB}$ & $2.98 \mathrm{~A}$ & $2.86 \mathrm{CD}$ & $0.72 \mathrm{BC}$ & $0.75 \mathrm{ABCD}$ & $3.58 \mathrm{AB}$ & $3.52 \mathrm{ABC}$ & $18.64 \mathrm{~A}$ & $17.90 \mathrm{CD}$ \\
\hline & $2 \mathrm{~g} / \mathrm{L}$ & $48.02 \mathrm{~A}$ & $47.49 \mathrm{~A}$ & $2.94 \mathrm{~A}$ & $3.02 \mathrm{~A}$ & $0.73 \mathrm{AB}$ & $0.78 \mathrm{AB}$ & $3.62 \mathrm{~A}$ & $3.66 \mathrm{~A}$ & $18.36 \mathrm{~A}$ & $18.88 \mathrm{~A}$ \\
\hline
\end{tabular}

* values having the same alphabetical letter (s) in common, do not significant different, using the revised L.S.D. test at 0.05 level of probability. 
Table 7: The interaction average values of some chemical analysis of artichoke head plants as affected by cultivars, NAA, GA3,6BA and seaweed extract during Fall season of 2016-2017 and 2017- 2018.

\begin{tabular}{|c|c|c|c|c|c|c|c|c|c|c|c|}
\hline & \multirow[t]{2}{*}{ Treatments } & \multicolumn{2}{|c|}{ Total chlorophyll $*$} & \multicolumn{2}{|c|}{$\mathrm{N}(\%)$} & \multicolumn{2}{|c|}{$\mathbf{P}(\%)$} & \multicolumn{2}{|c|}{$\mathrm{K}(\%)$} & \multicolumn{2}{|c|}{ Protein $(\%)$} \\
\hline & & $1^{\text {st }}$ season & $2^{\text {nd }}$ season & $1^{\text {st }}$ season & $2^{\text {nd }}$ season & $1^{\text {st }}$ season & $2^{\text {nd }}$ season & $1^{\text {st }}$ season & $2^{\text {nd }}$ season & $1^{\text {st }}$ season & $2^{\text {nd }}$ season \\
\hline \multirow{9}{*}{ 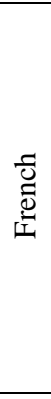 } & Control tap water & $45.47 \mathrm{abcd}$ & $45.17 \mathrm{f}$ & $2.59 \mathrm{~h}$ & $2.56 \mathrm{f}$ & $0.62 \mathrm{i}$ & $0.67 \mathrm{gh}$ & $3.18 \mathrm{j}$ & $3.43 \mathrm{de}$ & $16.17 \mathrm{~h}$ & $16.03 \mathrm{f}$ \\
\hline & NAA 25 ppm & $44.47 \mathrm{~cd}$ & 44.87 ef & $2.85 \mathrm{cdef}$ & $2.79 \mathrm{~cd}$ & $0.67 \mathrm{~h}$ & $0.69 \mathrm{fgh}$ & $3.24 \mathrm{ij}$ & $3.98 \mathrm{ab}$ & $17.79 \mathrm{def}$ & $17.43 \mathrm{~cd}$ \\
\hline & NAA 50 ppm & $47.37 \mathrm{ab}$ & $46.39 \mathrm{bcd}$ & $2.97 \mathrm{ab}$ & $2.92 \mathrm{ab}$ & 0.69 efgh & $0.71 \mathrm{fgh}$ & $3.23 \mathrm{ij}$ & $3.51 \mathrm{cde}$ & $18.54 \mathrm{ab}$ & $18.26 \mathrm{ab}$ \\
\hline & $\mathrm{GA}_{3} 30 \mathrm{ppm}$ & $47.20 \mathrm{ab}$ & 46.30 bcde & $2.79 \mathrm{ab}$ & $2.96 \mathrm{ab}$ & 0.72 bcde & 0.79 bcde & $3.29 \mathrm{hi}$ & $3.68 \mathrm{bcd}$ & $17.44 \mathrm{fg}$ & $18.49 \mathrm{ab}$ \\
\hline & $\mathrm{GA}_{3} 60 \mathrm{ppm}$ & $46.37 \mathrm{abcd}$ & $45.39 \mathrm{def}$ & $2.96 \mathrm{ab}$ & $2.79 \mathrm{~cd}$ & 0.72 bcde & 0.74 cdef & $3.47 \mathrm{def}$ & $3.65 \mathrm{~cd}$ & $18.48 \mathrm{abc}$ & $17.43 \mathrm{~cd}$ \\
\hline & 6BA $20 \mathrm{ppm}$ & $46.23 \mathrm{abcd}$ & $45.63 \mathrm{bcdef}$ & $2.84 \mathrm{def}$ & $2.73 \mathrm{de}$ & 0.71 bcde & $0.71 \mathrm{fgh}$ & 3.40 efg & $3.63 \mathrm{~cd}$ & 17.75 ef & $17.09 \mathrm{de}$ \\
\hline & 6BA $30 \mathrm{ppm}$ & $47.13 \mathrm{ab}$ & $46.70 \mathrm{bcd}$ & $2.96 \mathrm{ab}$ & $3.00 \mathrm{a}$ & 0.71 bcde & $0.72 \mathrm{efg}$ & $3.39 \mathrm{fgh}$ & $3.52 \mathrm{cde}$ & $18.52 \mathrm{ab}$ & $18.74 \mathrm{a}$ \\
\hline & Seaweed $1 \mathrm{~g} / \mathrm{L}$ & $47.20 \mathrm{ab}$ & $47.06 \mathrm{~b}$ & 2.92 abcde & $2.76 \mathrm{~d}$ & 0.70 defg & $0.74 \mathrm{cdef}$ & $3.56 \mathrm{abcd}$ & $3.76 \mathrm{bc}$ & 18.27 abcde & $17.26 \mathrm{~d}$ \\
\hline & Seaweed $2 \mathrm{~g} / \mathrm{L}$ & $48.03 \mathrm{a}$ & $47.06 \mathrm{~b}$ & $2.99 \mathrm{a}$ & $3.01 \mathrm{a}$ & $0.73 \mathrm{bc}$ & $0.69 \mathrm{fgh}$ & $3.57 \mathrm{abcd}$ & $4.15 \mathrm{a}$ & $18.70 \mathrm{a}$ & $18.84 \mathrm{a}$ \\
\hline \multirow{9}{*}{ 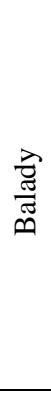 } & Control tap water & $45.33 \mathrm{bcd}$ & $44.78 \mathrm{f}$ & $2.52 \mathrm{~h}$ & $2.51 \mathrm{f}$ & $0.66 \mathrm{~h}$ & $0.64 \mathrm{~h}$ & $3.35 \mathrm{gh}$ & $3.10 \mathrm{~g}$ & $15.77 \mathrm{~h}$ & $15.66 \mathrm{f}$ \\
\hline & NAA 25 ppm & $44.33 \mathrm{~d}$ & $44.48 \mathrm{f}$ & $2.72 \mathrm{~g}$ & $2.62 \mathrm{ef}$ & $0.75 \mathrm{ab}$ & 0.73 defg & $3.51 \mathrm{cde}$ & $3.12 \mathrm{fg}$ & $17.00 \mathrm{~g}$ & 16.38 ef \\
\hline & NAA 50 ppm & $47.33 \mathrm{ab}$ & $46.50 \mathrm{bcd}$ & 2.88 bcdef & $2.94 \mathrm{ab}$ & $0.73 \mathrm{bc}$ & $0.80 \mathrm{abcd}$ & $3.50 \mathrm{cdef}$ & $3.41 \mathrm{def}$ & 17.98 bcdef & $18.38 \mathrm{ab}$ \\
\hline & $\mathrm{GA}_{3} 30 \mathrm{ppm}$ & $47.33 \mathrm{ab}$ & $46.96 \mathrm{bc}$ & $2.83 \mathrm{ef}$ & $2.96 \mathrm{ab}$ & $0.68 \mathrm{fgh}$ & 0.74 cdef & $3.55 \mathrm{bcd}$ & $3.38 \mathrm{defg}$ & $17.71 \mathrm{ef}$ & $18.48 \mathrm{ab}$ \\
\hline & $\mathrm{GA}_{3} 60 \mathrm{ppm}$ & $46.33 \mathrm{abcd}$ & $46.45 \mathrm{bcd}$ & $2.89 \mathrm{def}$ & $2.93 \mathrm{ab}$ & $0.68 \mathrm{fgh}$ & 0.73 defg & $3.58 \mathrm{abc}$ & 3.30 efg & 18.06abcdef & $18.31 \mathrm{ab}$ \\
\hline & 6BA $20 \mathrm{ppm}$ & $47.33 \mathrm{ab}$ & $45.51 \mathrm{cdef}$ & $2.85 \mathrm{cdef}$ & $2.85 \mathrm{bcd}$ & $0.74 \mathrm{ab}$ & 0.74 defg & $3.59 \mathrm{abc}$ & $3.23 \mathrm{efg}$ & 17.83 cdef & $17.83 \mathrm{bcd}$ \\
\hline & 6BA $30 \mathrm{ppm}$ & $46.33 \mathrm{abcd}$ & $47.01 \mathrm{~b}$ & 2.91abcde & $2.91 \mathrm{abc}$ & $0.77 \mathrm{a}$ & $0.82 \mathrm{ab}$ & $3.64 \mathrm{ab}$ & $3.43 \mathrm{de}$ & 18.20 abcde & $18.19 \mathrm{abc}$ \\
\hline & Seaweed $1 \mathrm{~g} / \mathrm{L}$ & $47.00 \mathrm{abc}$ & $47.06 \mathrm{~b}$ & $2.97 \mathrm{ab}$ & $3.03 \mathrm{a}$ & $0.74 \mathrm{ab}$ & $0.81 \mathrm{abc}$ & $3.59 \mathrm{abc}$ & $3.11 \mathrm{fg}$ & $18.56 \mathrm{ab}$ & $18.93 \mathrm{a}$ \\
\hline & Seaweed $2 \mathrm{~g} / \mathrm{L}$ & $48.00 \mathrm{a}$ & $48.68 \mathrm{a}$ & $2.95 a b c$ & $2.96 \mathrm{ab}$ & $0.78 \mathrm{a}$ & $0.87 \mathrm{a}$ & $3.66 \mathrm{a}$ & $3.43 \mathrm{de}$ & $18.43 \mathrm{ab}$ & $18.53 \mathrm{ab}$ \\
\hline
\end{tabular}

* values having the same alphabetical letter (s) in common, do not significant different, using the revised L.S.D. test at 0.05 level of probability. 
At the positive combined treatment (Balady cultivar and gibberellic acid at $60 \mathrm{ppm}$ ), the estimated percentages increase in number of early head/plant and number of early head /feddan were 70.55 and $64.78 \%$, respectively as an average of the two seasons, compared to the control treatment. The present results are in agreement with those reported by Calabrese et al. (1994), Okasha et al. (1997), and Soria et al. (2020) who showed significant differences among some tested cultivar and different spraying materials in their number of heads and earliness.

Moreover, the combined treatment of Balady cultivar and N-6-benzyle amino purine at $30 \mathrm{ppm}$ logged the highest mean values of total number of heads/plant and total number of head/feddan in the second season only and weight of edible part/ plant for both seasons. However, the combined treatment of Balady cultivar and seaweed extract at $2 \mathrm{~g} / \mathrm{L}$ recorded the highest mean values of total number of head /plant and total number of head /feddan in the first season only, compared to the other treatments. At the combined treatment (Balady cultivar and $\mathrm{N}$ 6-benzyle amino purine at $30 \mathrm{ppm}$ ), the estimated percentages increase in total edible weight /plant were 62.63 and $61.33 \%$, in comparison to the control treatment, in both seasons.

\section{Leaf chemical characteristics}

Data presented in Tables (6 and 7) indicated that the main effect of artichoke cultivars differed significantly in their leaf chemical characteristics. French cultivar showed higher mean values of nitrogen and protein in the first season. However, Balady cultivar exhibited higher mean values of potassium in both seasons. Meanwhile, the two cultivars are equal in total chlorophyll and phosphorus.

Regarding the main effect of the spraying treatments on leaf chemical characteristics of artichoke plants, the results reported in Tables (6 and 7) confirmed that spraying gibberellic acid, naphthalene acetic acid, N-6-benzyle amino purine and seaweed extract with all concentrations showed significant effects on total chlorophyll, nitrogen, phosphorus, potassium and protein contents, compared to control treatment, in both seasons. It is obvious that the best results were obtained from plants sprayed with seaweed extract at $2 \mathrm{~g} / \mathrm{l}$, followed by N-6-benzyle amino purine at $30 \mathrm{ppm}$ and gibberellic acid at $30 \mathrm{ppm}$, respectively, compared to the other treatments, in both seasons. using seaweed extract at $2 \mathrm{~g} / \mathrm{l}$ ), caused percentages increase in total chlorophyll, nitrogen, phosphorus, potassium and protein contents of (5.77 and \%), (14.84 and $21.77 \%)$, (14.06 and 20.00\%), (10.70 and $12.27 \%$ ) and (14.96 and $21.96 \%$ ), for the first and second seasons, respectively, compared to the control treatments.
The interaction effects between artichoke cultivars and the spraying treatments on leaf chemical characteristics of artichoke plants, are presented in (Table $6 \& 7$ ). The average values presented significant effects on the tested characteristics, during both seasons. The combined treatment of French cultivar and seaweed extract at $2 \mathrm{~g} / \mathrm{l}$, recorded the highest mean values of nitrogen and protein in both seasons, total chlorophyll in the first season and potassium in the second season. However, the combined treatment of Balady cultivar and seaweed extract at $2 \mathrm{~g} / \mathrm{l}$, reached the maximum for total chlorophyll and phosphorus in both seasons, potassium in the first season and protein, in the second season.

\section{REFERENCE}

Abd El-hamied, A. A., M. Kassim, T. and ElZoheiry, S. S. (2008). Effect of vernalization and gibberellic acid on earliness, total yield and quality of globe artichoke. Annals Agric. M.Sc., Moshtohor, 46 (4): 511-523.

A.O.A.C. (1975). Official methods of Analysis of the Association of Official Analytical

A.O.A.C. (1992). Official Methods of Analysis of the Association of Official Analytical Chemistis, $15^{\text {th }}$ Ed. Published by the association of Official Analytical Chemists III. North Nineteenth suite 210 Arlington, Virginia 2220/U.S.A.

Banowetz, G. M., Ammar, K.; Chen, D. D. (1999). Temperature effects on cytokinin accumulation and kernel mass in a dwarf wheat. Annals of Botany, 83(3): 303-307.

Basnitzki, Y.and D. Zohary (1987). A seed-planted cultivar of globe artichoke. Hort. Sci. 22 (4): $678-679$.

Basnitzki, Y and A. M. Mayer (1985). Germination of Cynara seeds; effect of light and temperature and function of the endosperm. Agronomie 5(6): 529-532.

Black, C. A. (1965). Methods of soil analysis. Part 2. Amer. Soc. Agric. [NC] Publisher. Inc. Madison, Wisconsin, USA.

Brengi, S. H. M. S. (2018). Growth, Yield and Chemical Composition of Okra as Affected by Three Types and Levels of Synthetic Cytokinins under High Temperature Conditions. Hort. Depart., Fac. of Agric, Daman Univ, Egypt. Alex. J. Agric. Sci. Vol. 63, No.6, pp. 365-372, 2018.

Bucan, L., S. Perica and S. Goreta (2000). Evaluation of artichoke (Cynara scolymus. L) cultivars in three growing seasons. Agric. Cons. Sci., 65 (1): 1- 8.

Calabrese, N., A. Elia and G. Sarli (1994) Yield and quality of new artichoke cultivars propagated by seed. Acta. Hort. (371): 189-193. 
De Malach, J. G., M. Sachs and R. Rotem (1976). Timing and optimal concentration of gibberellic acid treatments for forcing yield of globe artichoke (Cynara scolymus L.). Edition Minerva Medica, Turin, Italy, 633-642.

El-Baz, El-S., S.A. Foda and S.M. Mustapha (1979). Some studies on globe artichoke (Cynara scolymus, L.). Agric. Rese. Rev. Hort. 57 (3): 109-117

El-Greadly, N. H. M. (1994). Effect of some chemical substances on earliness, productivity and endogenous substances of globe artichoke, Ph.D. Thesis, Fac. of Agric., Cairo Univ.

El-Miniawy, S.M., M.E. Ragab., S.M. Youssef and A.A. Metwally (2014). Influence of foliar spraying of seaweed extract on growth, yield and quality of strawberry plants. Hort. Dept. Fac. of Agric. Univ. of Ain Shams, Egypt. 10 (2): pages 88-94.

Elia, A., F. Paolicelli and V. V. Bianco (1991). Effect of sowing date, plant density and nitrogen fertilizer on artichoke (Cynara scolymus L.): Preliminary results. Adv. Hort. Sci. 5(3): 119 - 122.

Evenhuis, B and P.W. Dewaard.(1980). Principles and practices in plant analysis. FAO .Soil Bull. 38(1):152-163.

FAO,

(2018).

http://www.fao.org/faostat/en/\#data/QC.

Fayed A.M. (1997). Evolution of some cultivars and mutants of cow pea (Vigna unguiculata L. Walp) under Kafr EL Sheikh condition M.Sci. Thesis. Fac . Agric., Kafr EL Sheikh, Tanta. University.

Gabr, S.M.; I. M. Ghoneim and H.A.EL-Khatib (2002). Effect of soil water matric potential on growth, yield and quality of two potato cultivars.J.Agric.Sci.Mansura Univ., 27(2): 1212-1220.

Gabr, S.M.and S.M.Sarg (1998). Response of some new potato cultivars grown in sandy soil to different nitrogen levels. Alex.J. Agric.43: 3342.

Gabrel, F., M. K., and Ali El, N. (2018). Effect of benzyl adenine and gibberellic acid on the vegetative growth and flowering of Chrysanthemum plant. Alexandria Journal of Agricultural Sciences, 63(1): 29-40.

Garcia, S.M., I.T. Firpo, F.S.L. Anido, E.L. Cointry (1999) Application of gibberellic acid in globe artichoke. Pesquisa Agropecuaria Brasileira 34 (5): 789-793.

George, E. F., M. A. Hall and G. J. De Klerk (2008). Plant Growth Regulators III: Gibberellins, Ethylene, Abscisic Acid, their Analogues and Inhibitors; Miscellaneous Compounds. In Plant propagation by tissue culture (pp. 227281).
Hamed, E.S.(2012). Effect of seaweed extract and compost treatments on growth, yield and quality of snap bean. phD Thesis, Hort. Dept., Fac. Agric., Ain Shams University.

Hammouda, F.M., M.M. Seif El-Nasr and A.A. Sahat (1993). Flavonoids of (Cynara scolymus L.) cultivated in Egypt. Plant Food for Human Nutrition 44 (2): 163 -169.

Hare, J. D., and Morgan, D. J. (1997). Mass-Priming Aphytis: Behavioral Improvement of InsectaryReared Biological Control Agents. Biological Control, 10(3), 207-214.

Hossain, F., Lettenmaier, D. P. (2006). Flood prediction in the future: Recognizing hydrologic issues in anticipation of the Global Precipitation Measurement mission. Water Resources Research, 42(11).

Ibrahim, T. M. A. (1980). Comparative morphology and anatomy of different artichokes cultivars and seed grown plant. Hort. Sci. 15 (3): 51.

Ibrahim, T.M.A. (2009). Studies on the development and production of globe artichoke (Cynara scolymus L.) under Sinai conditions. M.Sc. Thesis, Hort. Dept., Fac. Agric., Cairo Univ., Egypt.

Khan, W., U.P. Rayirath, S. Subramanian, M.N. Jithesh, P.Rayorath, D.M. Hodges, A.T. Critchley, J.S. Craigie,J. Norrie, B. Prithivira, (2009). Seaweed extracts as bio stimulants of plant growth and development, J. Plant Growth Regul., 28: 386-399.

Lin, T. C., S. H. Chuan and S. T. Horg (1991). Effect of chemicals on the enhancement of bolting in artichoke (Cynara scolymus L.). Bull. Taichung Distr. Agric. Improve. Stat., 32: 1115.

Mauromicale, G., A. Ierna (2000). Characteristics of heads of seed-grown globe artichoke (Cynara cardunculus L. var. scolymus (L.) Fiori) as affected by harvest period, sowing date and gibberellic acid. Agronomy 20 (2): 197-204.

Mauromicale, G., A. Ierna and V. Cavallaro (2000). Effects of vernalization and Gibberellic acid on bolting, harvest time and yield of seed-grown globe artichoke. Acta Hortic., 681: 243-250.

Mauromicale, G., P. Licandro., A. Ierna., N. Morello and G. Santoiemma (2003). Planning of Globe Artichoke plantlets production in nursery. Acta Hort., 660: 279-284.

Mc-Erlich, A. (1983): Globe artichoke varieties. New Zealand commercial grower. 35(4). (C.F. Hort. Abstr. 53(11): 7740.

Miccolis, V., A. Elia and V.V. Bianco (1990). Timing field production in a germplasm collection of artichoke (Cynara scolymus L.). Acta. Hort. 267: 153-161. 
Miguel, A., C. Baixauli, J. M. Aguilr, A. Giner, J. V. Maroto, S. Lopez, A. San Bautista and B. Pascual (2003). Gibberellic acid concentrations in seed propagated Artichoke. Acta Hort., 660: 167-172.

Okasha, KH.A., M.E. Ragab, H. El-Sayed Wahba, A.M. Razin, M.A. Abd-El-Salam (1997). Yield, head quality and some medicinal compounds of some new imported artichoke cultivars (Cynara scolymus L.). Zagazig Jour. of Agric. Res. 24 (1): 101- 115 .

Pandita, P.N., K. R. Ogra and K. Archana (1988). Evaluation of exotic cultivars for yield and chemical characters of globe artichokes (Cynara scolymus L.) in Kashmir Valley. Indian Jour. of Agric. Sci., 58 (9): 724 - 726.

Reddy, B.V.S, S. Ramesh, P.S. Reddy and A.A. Kumar (2009). Genetic enhancement for drought tolerance in sorghum. In: Janick, J. (Ed.), Plant Breeding Reviews. John Willey \& Sons, Inc. USA pp. 189-222.

Rodrigo, M.; J. Safon,; P.Lorenzo,; A Navarro,. and J.L Vaya, (1979). Agronomic and processing characteristic of globe artichoke cultivars. Influence of harvesting condition on yield and quality revistade -Agroquimica Y Tecnologia de Alimentos 19 (4): 498-512. (C.F. Hort. Abst. 50 (12): 740.

Saber, S., Y. A. Okdah and S. F. EL-Abd (2003). Gibberellin $\mathrm{GA}_{3}$ induced histological and histochemical alteration in the liver of Albino rats. Sci. Asia. 29: 327-331.
Saif Eldeen., U. M. M. M. B. Shokr and R. S. EL shotoury (2014). Effect of foliar spray with seaweeds extract and chitozan on earliness and productivity of gobe artichoke. Hort. Fac. Manus. Univ., Vol. 5 (7): 1197-1207.

Schrader, W.L., (1994). Growth regulator gives earlier harvest in artichokes. Calif. Agric. 48 (3), 29-32.

Schrader, W. L. and S. M. Keith (1997). Artichoke production in California. Division of Agriculture and Nate. Res., Univ. of California.

Schrader, W. L. (2005). Effects of plant growth regulators on heat stress in annual artichoke production. Acta Horticulturae, 681: 207-208.

Singh, D., P. K. Chhonker and B.S. Dwivedi (2005). Manual on soil plant and water analysis. West Ville Publishing House, New Delhi/ pp.200.

Soria, C., A. Baixauli., J.M. Giner and A.I. Nájera (2020). Productive and agronomic behavior of new cultivars and lines of seed propagated artichoke (Conference Paper) Experimental Center of Cajamarca, Cano., Cementerio nuevo s/n Ado. 194, Periportal, Valencia 46200, Spain Volume 1284, 6 July 2020, Pages 145-148.

Zaki, E. M., T. A. Abed., Gabal, M. R., M. M. ElAbagy, (1991). Effect of some growth regulators on growth, yield and chemical constituents of artichoke plants Cynara scolymus, L. Annals of Agricultural Science, Moshtohor. Egypt. 26(3):1939-1957. 


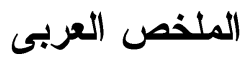

\section{نمو ومحصول وجودة صنفين من الخرشوف متآثراً بحمض الجبريلك، نفثالين حض الخليك، البنزيل امينو بيرورين ومستخلص الاعثاب البحرية}

\author{
سعيد محمد جبر، حسن أحمد الخطيب، ساري حسن برنجى، رمضان جمعة (براهيم \\ قسم البساتين - كلية الزر اعة- جامعة دمنهور .
}

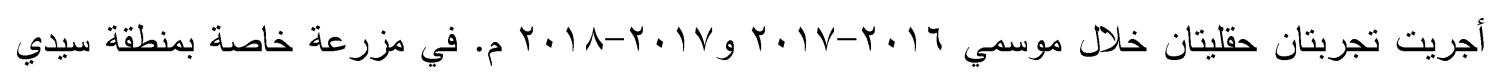
غازي مركز كفر الدوار - محافظة البحيرة.

تهدف هذه الدر اسة إلى اختبار استخدام الرش بحامض الجبرليك بتركيز (. ( و • ج جزء في المليون)، نفتالين

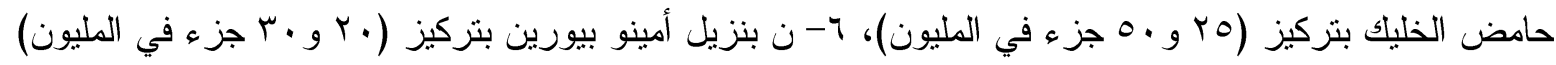
ومستخلص الأعشاب البحرية بتركيز (او r جرام / لتر).على نمو ومحصول وجودة صنفي الخرشوف (الفرنساوى و البلدي) تحت الظروف المصرية. أوضحت النتائج من خلال موسمي الزراعة أن الصنف الفرنساوى أعطى اعلى القيم لكلآ من ارتفاع النبات، عدد الأوراق، المساحة الورقية، عدد الفروع، الوزن الطازج والجاف، محتوى الأوراق من النيتزوجين والبروتين. بينما الصنف البلدي أعطى آعلى القيم لكلآ من عدد رؤوس الخرشوف المبكرة/ نبات وعدد الرؤوس المبكرة/ فدان، عدد الرؤوس الكلية للنبات و الفدان، ووزن الجزء الذي يؤكل من الرآس/نبات ومحتوى الأوراق من البوتاسيوم. أفادت الدر اسة أيضاً أن رش نبات الخرشوف بالمركبات السابقة وبكل التركيزات أعطت زيادة معنوية لمعظ صفات النمو الخضري و المحصول و المحتوى الكيماوي للأوراق مقارنة بمعاملة الكنترول وان كانت بدرجات مختلفة وفى هذا الصدد تفوق الرش بحامض الجبرليك في النمو الخضري و المحصول المبكر بينما تفوق الرش بمستخلص الأعشاب البحرية و ج-ن بنزيل آدينين في حالة المحصول الكلى و الصفات الكيماوية للأوراق. أشارت الدر اسة أن أفضل معاملة تداخلية هي زر اعة الصنف البلدي و الرش بمستخلص الأعشاب بتركز بجر ام / لتز أو الرش ن -7 بنزيل أدينين بتركيز • ب جزء في المليون حيث تعتبر المعاملة المثلى لإنتاج الخرشوف بكمية عالية وبجودة مرتفعة. 